\title{
CLASSES OF BAIRE FUNCTIONS
}

BY

\author{
GREGORY V. COX AND PAUL D. HUMKE
}

\begin{abstract}
Let $\mathcal{Q}$ and $\mathcal{P}$ denote the sets of approximately continuous and almost everywhere continuous functions, and $B_{1}(F)$ denote Baire's first class generated by $F$. The classes $B_{1}(\mathscr{Q}), B_{1}(\mathscr{P}), B_{1}(\mathscr{Q} \cap \mathscr{P})$, and Grande's class $\mathscr{Q} \mathscr{P}_{1}$ are investigated in some detail. Although Grande's question of whether $B_{1}(\mathbb{Q} \cap \mathcal{P})=B_{1}(Q) \cap$ $B_{1}(\mathbb{Q}) \cap \mathscr{Q} \mathcal{P}_{1}$ is not settled, we do show, among other results, that $\mathscr{Q} \mathscr{P}_{1} \subset B_{1}(\mathscr{P})$.
\end{abstract}

1. Introduction. In [G], Z. Grande considered the relationship between the classes of pointwise limits of approximately continuous functions, $B_{1}(\mathbb{Q})$, pointwise limits of almost everywhere continuous functions, $B_{1}(\mathcal{P})$, and pointwise limits of functions which are both approximately continuous and almost everywhere continuous, $B_{1}(\mathscr{Q} \cap \mathcal{P})$. After showing that $B_{1}(\mathscr{Q} \cap \mathscr{P}) \neq B_{1}(\mathscr{Q}) \cap B_{1}(\mathcal{P})$, Grande defined a new class of functions, which we (following [N]) denote $\mathscr{Q} \mathcal{P}_{1}$, and showed that

$$
B_{1}(\mathscr{Q} \cap \mathscr{P}) \subset B_{1}(\mathscr{Q}) \cap B_{1}(\mathscr{P}) \cap \mathscr{Q} \mathscr{P}_{1} .
$$

Grande then asked whether the inequality in (*) is indeed an equality. T. Nishiura [N] investigated Grande's question in the more general setting of real valued functions of $n$ real variables. Nishiura's approach was to study the topology of $\mathbb{Q} \cap \mathcal{P}$ in some detail and then glean from this more general study, results which would bear on Grande's question. The topology of $\mathscr{Q} \cap \mathscr{P}$ was introduced by $\mathbf{R}$. J. $\mathbf{O}^{\prime}$ Malley in the course of his investigations of approximate derivatives, $\left[\mathbf{O}_{\mathbf{1}}\right],\left[\mathbf{O}_{\mathbf{2}}\right]$. Indeed, O'Malley shows that the topology for $\mathscr{Q} \cap \mathcal{P}$ is the coarsest topology for which approximate derivatives are continuous. O’Malley, like Grande, was concerned with real valued functions of a single real variable. Nishiura's work led him to define a new class of functions, $\mathscr{Q} \mathscr{P}_{2}$, and subsequently he proved that

$$
B_{1}(\mathscr{Q} \cap \mathscr{P})=B_{1}(\mathscr{Q}) \cap B_{1}(\mathscr{P}) \cap \mathscr{Q} \mathscr{P}_{1} \cap \pm \mathscr{Q} \mathscr{P}_{2}
$$

where $\pm \mathscr{Q} \mathscr{P}_{2}$ consists of those functions in $\mathscr{Q} \mathscr{P}_{2}$ whose negatives are also in $\mathscr{Q} \mathscr{P}_{2}$. However, it is not clear whether $\pm \mathscr{Q} \mathscr{P}_{2}$ is essential in (**).

In this paper, we study the class $\mathscr{Q} \mathscr{P}_{1}$ in some detail, and although we do not settle Grande's question, we do provide some insight into the class $\mathscr{Q} \mathscr{P}_{1}$. In particular, we prove that $\mathscr{Q} \mathscr{P}_{1} \subset B_{1}(\mathcal{P})$.

In this paper, we deal only with real valued functions defined on $R^{n}$. As in the previous paragraph, if $\mathscr{F}$ is a class of functions, we let $B_{1}(\mathscr{F})$ (Baire 1 of $\mathscr{F}$ ) denote the class of those functions which are pointwise limits of functions in $\mathscr{F} ; B_{2}(\mathscr{F})=$ $B_{1}\left(B_{1}(\mathscr{F})\right)$ and so on. If $\mathscr{F}$ is the class of those functions which are continuous

Received by the editors May 23, 1980 and, in revised form, January 28, 1981.

1980 Mathematics Subject Classification. Primary 26A21; Secondary 26B05.

Key words and phrases. Approximately continuous, almost everywhere continuous, Baire function. 
relative to a topology on $R^{n}$, then there is a characterization of $B_{\alpha}(\mathscr{F})$ in terms of the Baire sets associated with that topology in the same way that the classical Baire functions are characterized by the usual Baire sets. That is, if $A$ and $M$ respectively denote the cozero and zero sets of the continuous functions, then $f \in B_{\alpha}(\mathscr{F})$ if and only if the preimage of every open set under $f$ is in the $\alpha$ th additive Baire class, e.g., $M_{\sigma}$ for $\alpha=1, A_{\delta \sigma}$ for $\alpha=2$. The three classes of functions which initiated this study are $\mathscr{P}, \mathbb{Q}$ and $\mathscr{Q} \cap \mathcal{P}$, and the latter two of these are the continuous functions for specific topologies. As a consequence, the Baire functions for $\mathscr{Q}$ and $\mathscr{Q} \cap \mathcal{P}$ are characterized by the associated Baire sets as described above. The approximately continuous functions are precisely those functions which are continuous with respect to the density topology. (The density topology consists of those measurable sets for which every point of the set is a point of full density of the set $[\mathbf{G W}]$.) In $\left[\mathbf{O}_{1}\right]$ and $[\mathbf{N}]$, O'Malley (for functions of a real variable) and Nishiura (for functions of $n$ real variables) show that $\mathscr{Q} \cap \mathscr{P}$ is likewise generated by a topology. This topology consists of those density open sets which can be decomposed as $G \cup Z$ where $G$ is Euclidean open and $Z$ is a nullset (i.e., $Z$ has Lebesgue measure zero). It should be noted that neither $\mathscr{Q}$ nor $\mathcal{Q} \cap \mathcal{P}$ is generated by a normal topology for $R$, and consequently, Borel sets and Baire sets are not the same for these topologies.

In addition to the characterizations of $B_{1}(\mathscr{Q})$ and $B_{1}(\mathscr{Q} \cap \mathcal{P})$ in terms of appropriate Baire sets, we will make use of the following two theorems, respectively due to D. Preiss [P] and D. Mauldin [M]. In these theorems, $C$ denoted the class of usual continuous functions.

TheOREM P. $A$ function $f \in B_{1}(\mathfrak{Q})$ if and only if $f \in B_{2}(\mathcal{C})$.

THEOREM M. A function $f \in B_{1}(\mathcal{P})$ if and only if there is a function $g \in B_{1}(\mathcal{C})$ such that $\{x: f(x) \neq g(x)\}$ is contained in an $F_{\sigma}$ nullset.

Finally, a function $f \in \mathbb{Q} \mathscr{P}_{1}$ if whenever $a<b$ and $U$ and $V$ are nonempty sets satisfying $U \subset\{x: f(x)<a\}$ and $V \subset\{x: f(x)>b\}$, then $U \cup V \not \subset \Delta(\bar{U} \cap \bar{V})$; the bar denoted Euclidean closure and $\Delta(X)=\left\{x: d_{x}^{+}(X)>0\right\}$, the set of points of positive upper density of $X$. Although this is not the original definition of $\mathscr{Q} \mathcal{P}_{1}$, it is an equivalent definition (cf. $[\mathbf{C H}]$ ) which we find more workable.

2. Algebraic and topological properties. In this section, we show that all of the classes of functions which arise in this study have the following algebraic and topological properties:

(I) The class is a vector space over $R$.

(II) The class is closed under uniform convergence.

(III) If $f$ is a member of the class, then $f$ is the uniform limit of a sequence of simple functions (discrete ranges) from the class.

Lemma 2.1. The class, $B_{1}(\mathcal{Q})$, has each of properties (I), (II) and (III).

Proof. Since $B_{1}(\mathscr{Q})=B_{2}(\mathcal{C})$, these results are classical.

LEMMA 2.2. The class, $B_{1}(\mathcal{P})$, has each of properties (I), (II) and (III). 
Proof. The proof of (I) is obvious. To prove (II), let $\left\{f_{n}\right\} \subset B_{1}(\mathcal{P})$ and suppose $\left\{f_{n}\right\}$ converges uniformly to $f$. As $f_{n} \in B_{1}(\mathscr{P})$ for each $n$, we can apply Theorem M to obtain a $g_{n} \in B_{1}(\mathcal{C})$ such that $f_{n}(x)=g_{n}(x)$ except on an $F_{\sigma}$ nullset, $E_{n}$. Let $A=R^{m}-\cup_{n=1}^{\infty} E_{n}$. Then, for each $n, g_{n}=f_{n}$ on $A, g_{n} / A$ is a Baire 1 function on $A$, and $g_{n} / A$ converges uniformly to $f / A$. It follows that $f / A$ is a Baire 1 function on $A$ and as $A$ is a $G_{\delta}$ (multiplicative of Class 1 ), $f / A$ can be extended to a function $f^{*}$ which is $B_{1}(\mathcal{C})$ on $R^{m}[\mathbf{K}]$. However, $f(x)=f^{*}(x)$ on $A$ and it follows from Theorem M that $f \in B_{1}(\mathcal{P})$.

In order to prove (III), let $f \in B_{1}(\mathcal{P})$. Then there is a function $g \in B_{1}(\mathcal{C})$ such that $f(x)=g(x)$ except on an $F_{\sigma}$ nullset, $E$. As $g \in B_{1}(e)$ there is a sequence of simple functions $\left\{g_{n}\right\} \subset B_{1}(\mathcal{C})$ such that $\left|g_{n}(x)-g(x)\right|<1 / n$ for every $x$ and every $n$. Let $E_{n, i}=\{x \in E: i \leqslant n f(x)<i+1\}$ and define

$$
f_{n}(x)= \begin{cases}g_{n}(x) & \text { if } x \in E \\ i / n & \text { if } x \in E_{n, i} ; i=0, \pm 1, \pm 2, \ldots\end{cases}
$$

Then, $f_{n}$ is a simple function and as $f_{n}$ differs from $g_{n}$ on a subset of $E, f_{n} \in B_{1}(\mathcal{P})$. Further, $\left|f_{n}(x)-f(x)\right|<1 / n$ for every $x$, and consequently, $\left\{f_{n}\right\}$ converges uniformly to $f$.

LEMma 2.3. The class, $B_{1}(\mathscr{P} \cap \mathcal{P})$, has each of properties (I), (II) and (III).

Proof. Since $\mathscr{Q} \cap \mathcal{P}$ has property (I) (see [N]), so does $B_{1}(\mathscr{Q} \cap \mathscr{P})$. To see that $B_{1}(Q \cap \mathcal{P})$ has property (II), one can use the topology described in $[\mathbf{N}]$ and mimic the proof of Theorem 2, p. 386 of $[\mathbf{K}]$. To see that $B_{1}(Q \cap \cap \mathcal{P})$ has property (III), one again is able to adapt Kuratowski's work. In particular, the analogue of Theorem 1, p. 350 of [K] is true. That is, if $B_{1}$ and $B_{2}$ are $M_{\sigma}$ sets (in the topology of [N]), there exist $M_{\sigma}$ sets $A_{1}$ and $A_{2}$ such that $A_{1} \subset B_{1}, A_{2} \subset B_{2}, A_{1} \cap A_{2}=\varnothing$, and $A_{1} \cup A_{2}=B_{1} \cup B_{2}$. Moreover, if $B_{1} \cup B_{2}$ is the whole space, then $A_{1}$ and $A_{2}$ are $A_{\delta}$ sets. We then obtain as a corollary that if $A$ and $B$ are $A_{\delta}$ sets such that $A \cap B=\varnothing$, then there exists a set $A^{*}$ which is both $A_{\delta}$ and $M_{\sigma}$ and such that $A \subset A^{*}$ and $A^{*} \cap B=\varnothing$. We omit the proofs as they exactly follow those given in [K]. From these results, one can modify Theorem 3, p. 388 of [K] to conclude that $B_{1}(\mathbb{P} \cap \mathcal{P})$ has property (III).

Lemma 2.4. Suppose that $f \in \mathscr{Q P}_{1}, \varepsilon>0$, and $p \in R$. There exists $g \in B_{1}(\mathcal{C})$ such that

(1) $\sup |g(x)-p| \leqslant \varepsilon$, and

(2) $\{x: f(x)=g(x)\}$ lies in an $F_{\sigma}$ nullset.

Proof. We let $M_{0}=R^{n}$ and $N_{0}=\varnothing$. Since $f \in \mathscr{Q} \mathscr{P}_{1}$, we can choose $y_{0}$ satisfying $\left|p-y_{0}\right|<\varepsilon$ and such that $f^{-1}\left(y_{0}\right) \backslash N_{0}$ is nowhere dense (possibly empty) in $M_{0} \backslash N_{0}$.

Suppose that sets $M_{\alpha}, N_{\alpha}$, and points $y_{\alpha}$ have defined for every $\alpha<\beta$. If $\beta$ is a limit ordinal, define $M_{\beta}=\bigcap_{\alpha<\beta} M_{\alpha}$; otherwise define

$$
M_{\beta}=M_{\beta-1} \cap \overline{f^{-1}\left(y_{\beta-\alpha}\right) N_{\beta-1}} \text {. }
$$


In any case, $N_{\beta}=\left\{x \in M_{\beta}: d_{x}^{+}\left(M_{\beta}\right)<1\right\}$ and $y_{\beta}$ is any point satisfying $\left|p-y_{\beta}\right|$ $<\varepsilon$ and $f^{-1}\left(y_{\beta}\right) \backslash N_{\beta}$ is nowhere dense (possibly empty) in $\overline{M_{\beta} \backslash N_{\beta}}$. Notice that $N_{\beta}$ is an $F_{\sigma}$ nullset and that, unless $\left|M_{\beta}\right|=0,\left|M_{\beta} \backslash M_{\beta+1}\right|>0$.

There exists $\beta^{*}<\omega_{1}$ such that $\left|M_{\beta^{*}}\right|=0$. If $x \in M_{\beta^{*}}$, define $g(x)=y_{\beta^{*}}$. If $x \notin M_{\beta^{*}}$, there exists a first (nonlimit) ordinal $\alpha+1$ for which $x \in M_{\alpha+1}^{c}$, and we define $g(x)=y_{\alpha}$.

Since the range of $g$ is countable, and $g^{-1}\left(y_{\alpha}\right)=M_{\alpha+1}^{c} \cap\left(\cap_{\sigma \leqslant \alpha} M_{\alpha}\right)$ (unless $\alpha=\beta^{*}$, in which case $\left.g^{-1}\left(y_{\beta^{*}}\right)=M_{\beta^{*}}\right)$, then preimages of open sets are $F_{\sigma}$ sets and so $g \in B_{1}(e)$.

We let $N=M_{\beta^{*}} \cup\left(\cup_{\alpha<\beta^{*}} N_{\alpha}\right)$, and $N$ is an $F_{\sigma}$ nullset for which we claim that $\{x: f(x)=g(x)\} \subset N$. This is because for any $x$, either $x \in M_{\beta^{*}} \subset N$, or $x \in$ $M_{\alpha+1}^{c} \cap\left(\cap_{\sigma \leqslant \alpha} M_{\sigma}\right)$ for some $\alpha$. Now, if $f(x)=g(x)=y_{\alpha}$, then since $x \notin M_{\alpha+1}$, $x \notin \overline{f^{-1}\left(y_{\alpha}\right) \backslash N_{\alpha}}$, so $x \in N_{\alpha} \subset N$.

Lemma 2.5. If $h \in \mathbb{Q} \mathscr{P}_{1}$ and $a$ is a number such that $\{x: h(x)=a\}$ lies in an $F_{\sigma}$ nullset, then if $U$ and $V$ are nonempty sets satisfying the conditions $U \subset\{x$ : $h(x) \leqslant a\}$ and $V \subset\{x: h(x)>a\}, U \cup V \not \subset \Delta(\bar{U} \cap \bar{V})$.

Proof. Suppose that $a$ is a number and $U$ and $V$ are nonempty sets such that $U \subset\{x: h(x) \leqslant a\}$ and $V \subset\{x: h(x)>a\}$ and $U \cup V \subset \Delta(\bar{U} \cap \bar{V})$. In this case, we can assume that $U \cup V=\Delta(\bar{U} \cap \bar{V})$, since if $x \in \Delta(\bar{U} \cap \bar{V})$, either $h(x) \leqslant a$ or $h(x)>a$. Now, since $h^{-1}(a)$ lies in an $F_{\sigma}$ nullset, and $\bar{U} \cap \bar{V}$ has positive measure in each relative open set, then $h^{-1}(a)$ is of the first category in $\bar{U} \cap \bar{V}$, and, in fact, $h^{-1}(a)$ is of the first category in $\Delta(\bar{U} \cap \bar{V})$ since $\Delta(\bar{U} \cap \bar{V})$ is residual in $\bar{U} \cap \bar{V}$.

Either there exists $c>a$ such that $\{x: h(x)>c\} \cap V$ is somewhere dense in $\Delta(\bar{U} \cap \bar{V})$, or there exists $c<a$ such that $\{x: h(x)<c\} \cap U$ is somewhere dense in $\Delta(\bar{U} \cap \bar{V})$. In either case, we easily conclude that $h \notin \mathbb{Q} \mathscr{P}_{1}$.

Lemma 2.6. The class, $\mathbb{Q} \mathscr{P}_{1}$, has each of the properties (I), (II), and (III).

Proof. The only nontrivial part in showing that property (I) is satisfied is to show that sums of $\mathscr{Q} \mathscr{P}_{1}$ functions are $\mathscr{Q} \mathscr{P}_{1}$ functions. Suppose then that $f+g \notin$ $\mathscr{Q} \mathscr{P}_{1}$ while $f \in \mathscr{Q} \mathscr{P}_{1}$ and $g \in \mathbb{Q} \mathscr{P}_{1}$. Then we find $a<b$ and $\varnothing \neq U \subset\{x$ : $f(x)+g(x)<a\}$ and $\varnothing \neq V \subset\{x: f(x)+g(x)>b\}$ such that $U \cup V \subset$ $\Delta(\bar{U} \cap \bar{V})$. Then, $\bar{U}=\bar{V}$. Let $\varepsilon=(b-a) / 2$. There exists a number $y$ such that $\bar{V} \cap f^{-1}[(y-\varepsilon / 3, y+\varepsilon / 3)]$ is dense in some nonempty, relatively open subset $I$ of $\bar{V}$. Next, there is a number $z$ such that $I \cap g^{-1}[(z-\varepsilon / 3, z+\varepsilon / 3)]$ is dense in some nonempty, relatively open subset $I^{*}$ of $I$. Now, either $y+z+\varepsilon \leqslant b$ or $y+z-\varepsilon \geqslant a$, and we suppose the former. Since $f \in \mathbb{Q} \mathscr{P}_{1}, V=\Delta(\bar{V}), \varnothing \neq V$, and $I^{*}$ is a nonempty relatively open subset of $\bar{V}$, only a subset of $V \cap I^{*}$ which is nowhere dense in $I^{*}$ can be mapped by $f$ above $y+\varepsilon / 2$. And only a subset of $V \cap I^{*}$ which is nowhere dense in $I^{*}$ can be mapped by $g$ above $z+\varepsilon / 2$. Yet every point of $V$ is mapped by $f+g$ above $y+z+\varepsilon$, and we have a contradiction.

The proof that $\mathscr{Q} \mathscr{P}_{1}$ has property (II) is trivial. To show that $\mathscr{Q} \mathscr{P}_{1}$ has property (III), we consider $f \in \mathbb{Q} \mathscr{P}_{1}$ and a fixed positive integer $n$, and we describe a simple 
function, $s$, for which $\sup |f(x)-s(x)|<2 / n$. Let $p=\{m / n: \quad m=$ $0, \pm 1, \pm 2, \ldots\}, \varepsilon=1 /(3 n)$, and for each $p \in P$, apply Lemma 2.4 to get $g=g_{p}$. For brevity, let $p^{+}$denote $p+1 / n$, and $p-$ denote $p-1 / n$. For each $x$, there is a unique $p \in P$ such that $g_{p}(x)<f(x) \leqslant g_{p^{+}}(x)$; define $s(x)=p^{+}$.

To show that $s \in \mathbb{Q} \mathscr{P}_{1}$ let $a<b$ and consider the following cases: Case 1. There exists $p \in P$ such that $a \leqslant p \leqslant b$. Case 2 . There exist adjacent points $p$ and $p+$ in $P$ such that $p<a<b<p+$.

Case 1. If $\varnothing \neq U \subset\{x: s(x)<a\}$ and $\varnothing \neq V \subset\{x: s(x)>b\}$, then in fact, $U \subset\{x: s(x) \leqslant p-\}$ and $V \subset\{x: s(x)>p\}$. In other words, $U \subset\{x: f(x) \leqslant$ $\left.g_{p-}(x)\right\}$ and $V \subset\left\{x: f(x)>g_{p}(x)\right\}$. Now, there exist numbers $a^{\prime}$ and $b^{\prime}$ such that for every $x, g_{p}(x)<a^{\prime}<b^{\prime}<g_{p}(x)$. This means that $U \subset\left\{x: f(x)<a^{\prime}\right\}$ and $V \subset\left\{x: f(x)>b^{\prime}\right\}$, so $U \cup V \not \subset \Delta(\bar{U} \cap \bar{V})$.

Case 2. If $\varnothing \neq U \subset\{x: s(x)<a\}$ and $\varnothing \neq V \subset\{x: s(x)>b\}$, then in fact $U \subset\left\{x: f(x) \leqslant g_{p}(x)\right\}=\left\{x: f(x)-g_{p}(x) \leqslant 0\right\}$, and $V \subset\left\{x: f(x)>g_{p}(x)\right\}=$ $\left\{x: f(x)-g_{p}(x)>0\right\}$. We know that $h=f-g_{p}$ belongs to $\mathscr{Q} \mathscr{P}_{1}$ from the first part of this lemma and the fact that $B_{1}(\mathcal{C}) \subset B_{1}(\mathbb{Q} \cap \mathcal{P}) \cap \mathbb{Q} \mathscr{P}_{1}$. We also know that $\left\{x: f(x)-g_{p}(x)=0\right\}=\left\{x: f(x)=g_{p}(x)\right\}$ lies in an $F_{\sigma}$ nullset. Thus, by Lemma 2.5 (with $a=0$ ), $U \cup V \not \subset \Delta(\bar{U} \cap \bar{V})$.

LEMma 2.7. The class, $B_{1}(\mathscr{Q}) \cap B_{1}(\mathscr{P}) \cap \mathscr{Q} \mathscr{P}_{1}$, has each of properties (I), (II) and (III).

Proof. Let $f \in B_{1}(\mathscr{Q}) \cap B_{1}(\mathscr{P}) \cap \mathscr{Q} \mathscr{P}_{1}$. By Theorem $M$ there is a $g \in B_{1}(\mathcal{C})$ such that $\{x: f(x) \neq g(x)\}$ lies in an $F_{\sigma}$ nullset. Let $h=f-g$. Since $B_{1}(\mathcal{C}) \subset$ $B_{1}(\mathscr{Q} \cap \mathcal{P})$, we have by (*) and Lemmas 2.1, 2.2, and 2.6 that $h \in B_{1}(\mathscr{Q}) \cap B_{1}(\mathscr{P})$ $\cap \mathscr{Q} \mathscr{P}_{1}$. There is an integer-valued function $s \in B_{1}(\mathfrak{Q})$ such that $|h(x)-s(x)|<1$ for all $x$. Let $\sigma(x)=s(x)$ when $|s(x)| \geqslant 2$ and $\sigma(x)=0$ when $|s(x)| \leqslant 1$. Then $\{x$ : $\sigma(x) \neq 0\} \subset\{x: h(x) \neq 0\}$. Hence, $\sigma$ is integer-valued, $\sigma \in B_{1}(\mathscr{Q}) \cap B_{1}(\mathcal{P})$, $\sigma^{-1}[(-2,2)]=\sigma^{-1}(0)$, and $|h(x)-\sigma(x)|<3$ for all $x$. Suppose $\sigma \notin \mathbb{Q} \mathscr{P}_{1}$. Then we can find $a<b, \varnothing \neq U \subset\{x: \sigma(x)<a\}, \varnothing \neq V \subset\{x: \sigma(x)>b\}$ such that $U \cup V \subset \Delta(\bar{U} \cap \bar{V})$. Either $a<0$ or $b>0$ and for definiteness we suppose $b>0$. Since $\{x: h(x) \neq 0\}$ is a nullset and $\bar{U} \cap \bar{V}=\overline{\Delta(\bar{U} \cap \bar{V})} \neq \varnothing$, the set $W=\Delta(\bar{U} \cap \bar{V}) \cap\{x: h(x)=0\}$ is dense in $\Delta(\bar{U} \cap \bar{V})$. For each $x \in V$, we have $2 \leqslant \sigma(x)=s(x)$. Hence, $V \subset\{x: h(x) \geqslant 1\}$. Since $\bar{W}=\overline{\Delta(\bar{U} \cap \bar{V})}=\bar{V}$, we have $W \cup V \subset \Delta(\bar{W} \cap \bar{V})$ and $W \neq \varnothing \neq V$. This is not possible, however, because $h \in \mathscr{Q} \mathscr{P}_{1}$. We have shown that $\sigma \in \mathbb{Q} \mathscr{P}_{1}$ and the lemma now follows.

RemarK. An inspection of the proof of Lemma 2.7 reveals that the class, $B_{1}(\mathcal{Q}) \cap B_{1}(\mathcal{P})$, has each of properties (I), (II) and (III).

We now combine Lemmas 2.1-2.3, 2.6 and 2.7 to get the following.

THEOREM 2.8. The following classes have properties (I), (II) and (III): $B_{1}(\mathbb{Q})$, $B_{1}(\mathscr{P}), \mathscr{Q} \mathscr{P}_{1}, B_{1}(\mathscr{P} \cap \mathcal{P})$, and $B_{1}(\mathscr{Q}) \cap B_{1}(\mathcal{P}) \cap \mathscr{Q P} \mathscr{P}_{1}$.

REMARK. As remarked above, $B_{1}(\mathscr{Q}) \cap B_{1}(\mathscr{P})$ has properties (I), (II) and (III). In $\S 3$, we show that $\mathscr{Q} \mathscr{P}_{1} \subset B_{1}(\mathcal{P})$, so in fact, $B_{1}(\mathscr{P}) \cap \mathscr{Q} \mathscr{P}_{1}=\mathscr{Q} \mathscr{P}_{1}$ has (I), (II) and (III), and $B_{1}(\mathscr{Q}) \cap \mathscr{Q} \mathscr{P}_{1}=B_{1}(\mathscr{Q}) \cap B_{1}(\mathscr{P}) \cap \mathscr{Q} \mathscr{P}_{1}$ has (I), (II) and (III). 
3. More on $B_{1}(\mathscr{P})$ and $Q \mathscr{P}_{1}$. The significance of the topological properties discussed in $\S 2$ is that we may limit our attention to simple functions (and, indeed, to characteristic functions). We begin with a theorem which appears quite special, but subsequently show that the hypothesis of this theorem locally characterizes those characteristic functions which are in $Q_{\mathcal{P}} \mathscr{P}_{1}$ in a fundamental way.

THEOREM 3.1. Suppose that $K$ is a set such that either $\Delta \bar{K} \subset K$ or $\Delta \bar{K} \cap K=\varnothing$. Then $\chi_{K} \in B_{1}(\mathscr{P}) \cap \mathscr{Q P} \mathscr{P}_{1}$.

Proof. Suppose, first, that $\Delta \bar{K} \subset K$. We compare the function $f=\chi_{K}$ with the function $g=\chi_{\bar{K}}$, and notice that $\{x: f(x) \neq g(x)\} \subset\left\{x \in \bar{K}: d_{x}^{+}(\bar{K})<1\right\}$. As the latter is an $F_{o}$ nullset, Theorem $M$ implies that $\chi_{K} \in B_{1}(\mathcal{P})$. To show that $\chi_{K} \in Q \mathscr{P}_{1}$, it suffices to consider nonempty sets $U \subset K^{c}$ and $V \subset K$. Since $V \subset K, \Delta(\bar{U} \cap \bar{V}) \subset \Delta \bar{K} \subset K$. Now, since $U \subset K^{c}, U$ cannot be a subset of $\Delta(\bar{U} \cap \bar{V}) \subset K$. Thus, $U \cup V \ell \Delta(\bar{U} \cap \bar{V})$.

Now suppose that $\Delta \bar{K} \cap K=\varnothing$. We compare the function $f=\chi_{K}$, with the function $g$, which is identically zero. As before, $\{x: f(x) \neq g(x)\}$ lies in an $F_{\sigma}$ nullset, and so $\chi_{K} \in B_{1}(\mathscr{P})$. To show that $\chi_{K} \in \mathbb{Q} \mathscr{P}_{1}$, we again consider $U \subset K^{c}$ and $V \subset K$. Now $\bar{V} \subset \bar{K}$, and consequently, $\Delta(\bar{U} \cap \bar{V}) \subset \Delta \bar{K}$; however $V \subset K$ and $\Delta \bar{K} \cap K=\varnothing$. This entails that $V \not \Delta(\bar{U} \cap \bar{V})$ and so $\chi_{K} \in \mathbb{Q} \mathscr{P}_{1}$.

Our next goal is to start with a characteristic function, $\chi_{K} \in \mathbb{Q} \mathscr{P}_{1}$, and decompose $K$ into subsets which satisfy the hypothesis of Theorem 3.1.

Lemma 3.2. Suppose that $G$ is an open set and that $K$ is a set such that if $F$ is a closed subset of $G, \Delta \overline{(F \cap K)} \subset F \cap K$. Then $\chi_{K \cap G} \in B_{1}(\mathscr{P})$.

Proof. Express $G$ as $G=F_{1} \cup F_{2} \cup \cdots$, where $F_{i+1}$ is the closure of an open set containing $F_{i}(i=1,2, \ldots)$. For each $n$, Theorem 3.1 guarantees that there is a sequence, $\left\{f_{i}^{n}: i=1,2, \ldots\right\}$ from $\mathcal{P}$ that converges to $\chi_{K \cap F_{n}}$. Now, let $\rho$ denote Euclidean distance, and define weighting functions $U_{i}^{n}$ as follows:

$$
U_{i}^{n}(x)=\min \left\{i \cdot \rho\left(x, F_{n}\right), 1\right\} .
$$

Each $U_{i}^{n}$ is a continuous function whose range is $[0,1]$, and for each $n,\left\{U_{i}^{n}\right.$ : $i=1,2, \ldots\}$ converges to $\chi_{F_{n}^{c}}$. Now define

$$
\begin{aligned}
& h_{1}=U_{1}^{1} \cdot f_{2}^{2}+\left(1-U_{1}^{1}\right) \cdot f_{2}^{1}, \\
& h_{2}=U_{1}^{2} \cdot f_{3}^{3}+\left(1-U_{1}^{2}\right)\left(U_{2}^{1} \cdot f_{3}^{2}+\left(1-U_{2}^{1}\right) \cdot f_{3}^{1}\right), \\
& h_{3}=U_{1}^{3} \cdot f_{4}^{4}+\left(1-U_{1}^{3}\right)\left(U_{2}^{2} \cdot f_{4}^{3}+\left(1-U_{2}^{2}\right)\left(U_{3}^{1} \cdot f_{4}^{2}+\left(1-U_{3}^{1}\right) \cdot f_{4}^{1}\right)\right),
\end{aligned}
$$

and so on.

As each $f_{i}^{n} \in \mathscr{P}$ and each $U_{i}^{n}$ is continuous, each $h_{n} \in \mathscr{P}$. Now, if $x \in G$, there is a first $n$ such that $x \in F_{n}$, and consequently, if $m \geqslant n, U_{i}^{m}(x)=0$ for each $i$. There is an $i_{n-1}$ such that if $j \geqslant i_{n-1}$, then $U_{j}^{n-1}(x)=1$. For notational purposes we have let $U_{i}^{0}(x)=1$ and $i_{0}=0$. If $s=\max \left\{n, i_{n-1}+n-2\right\}$, then $k>s$ implies $h_{k}(x)=f_{k+1}^{n}(x)$. Hence, as $k \rightarrow \infty, h_{k}(x) \rightarrow \chi_{K \cap F_{n}}(x)=\chi_{K \cap G}(x)$. This completes the proof. 
THEOREM 3.3. Suppose that $\chi_{K} \in \mathscr{Q P}_{\mathcal{P}_{1}}$, and let $G$ and $H$ denote the maximal open sets missing $\Delta \bar{K} \backslash K$ and $\Delta \bar{K} \cap K$ respectively. Then both $\chi_{K \cap G}$ and $\chi_{K \cap H}$ are in $B_{1}(\mathcal{P})$, and consequently, letting $I=G \cup H, \chi_{K \cap I} \in B_{1}(\mathcal{P})$.

Proof. The conclusion concerning $\chi_{K \cap G}$ follows from Lemma 3.2. Since $\Delta \overline{(H \cap K)} \cap(H \cap K)=\varnothing$, we apply Theorem 3.1 to conclude that $\chi_{K \cap H} \in$ $B_{1}(\mathcal{P})$.

THEOREM 3.4. Suppose that $\chi_{K} \in \mathcal{Q} \mathscr{P}_{1}$, and let $G$ and $H$ denote the maximal open sets missing $\Delta \bar{K} \backslash K$ and $\Delta \bar{K} \cap K$ respectively. If $|K|>0$, then $|\bar{K} \cap(G \cup H)|>$ 0 .

Proof. Let $W$ denote the maximal open set missing $\Delta \bar{K}$. Now, $|\bar{K}|=|\bar{K} \backslash W|>$ 0 , and as $\chi_{K} \in \mathbb{Q} \mathscr{P}_{1}, \bar{K} \backslash W$ intersects either $G$ or $H$. This intersection must have positive measure.

Now, starting with a set $K$ whose characteristic function is $\mathscr{Q} \mathscr{P}_{1}$, we extract open sets $G$ and $H$ as in Theorems 3.3 and 3.4. Then, instead of working with $K$ itself, we work with $K_{1}=K \backslash(G \cup H)$. We repeat this process inductively until we have a set whose closure is a nullset, and according to Theorem 3.4, this situation occurs before we reach $\omega_{1}$. There are still some technical difficulties to overcome before we can put this machinery to use. In particular, although Theorem 3.3 guarantees a $B_{1}(\mathscr{P})$ function at each inductive step, we need to show that in the limiting case, we still have a $B_{1}(\mathcal{P})$ function. This is the charge of the following two convergence results.

THEOREM 3.5. Suppose that $K$ is a set and that $\left\{W_{n}: n=1,2, \ldots\right\}$ is an increasing sequence of open sets with union $W$. If, for each $n, \chi_{K \cap W_{n}} \in B_{1}(\mathscr{P})$, then $\chi_{K \cap W} \in B_{1}(\mathcal{P})$.

Proof. There is an increasing sequence of open sets, $\left\{G_{n}: n=1,2, \ldots\right\}$, such that $\bar{G}_{n} \subset G_{n+1}$ for every $n$, every $G_{n}$ is contained in some $W_{m}$, and $\cup G_{n}=W$. For convenience, let $G_{0}=\varnothing$. Now, for each $n>0$, one can find an increasing sequence of closed sets, $\left\{F_{i}^{n}: i=1,2, \ldots\right\}$, such that $\bar{G}_{n-1} \subset F_{1}^{n}$, and $\cup_{i<\omega_{0}} F_{i}^{n}$ $=G_{n}$. For each set $F_{i}^{n}$, define a weighting function, $U_{i}^{n}$, as follows:

$$
U_{i}^{n}=\max \left\{1-\rho\left(x, F_{i}^{n}\right) / \rho\left(F_{i}^{n}, G_{n}^{c}\right), 0\right\} .
$$

As each set, $G_{n}$, is contained in some $W_{m}$, and since $\chi_{G_{n}} \in B_{1}(\bigodot)$, it follows that $\chi_{K \cap G_{n}} \in B_{1}(\mathcal{P})$ for each $n$. Consequently, for every $n$, there is a sequence, $\left\{f_{i}^{n}\right.$ : $i=1,2, \ldots\}$ from $\mathscr{P}$ which converges to $\chi_{K \cap G_{n}}$. Define a new sequence of functions by

$$
g_{i}=U_{1}^{1} \cdot f_{1}^{1}+U_{i}^{2} \cdot\left(1-U_{1}^{1}\right) \cdot f_{i}^{2}+\cdots+U_{i}^{i} \cdot\left(1-U_{i}^{i-1}\right) \cdot f_{i}^{i} .
$$

As each $U_{i}^{n}$ is continuous, and each $f_{i}^{n} \in \mathscr{P}$, each $g_{i} \in \mathcal{P}$. Furthermore, if $x \in W$, then there is a first lexicographically ordered pair, $(n, i)$, such that $x \in F_{i}^{n}$, and it follows that $x \in F_{j}^{n}$ for all $j \geqslant i$. But then, if $j \geqslant \max \{n, i\}, g_{j}(x)=f_{j}^{n}(x)$, and the latter converges, with $j$, to $\chi_{K \cap G_{n}}(x)=\chi_{K \cap G}(x)$. If $x \notin W$, then $g_{i}(x)=0$ for each $i$, and this observation completes the proof. 
Corollary 3.6. Suppose that $K$ is a set, $\lambda<\omega_{1}$, and that $\left\{W_{\alpha}: \alpha<\lambda\right\}$ is an increasing (possibly transfinite) sequence of open sets with union $W$. If, for each $\alpha$, $\chi_{K \cap W_{\alpha}} \in B_{1}(\mathscr{P})$, then $\chi_{K \cap W} \in B_{1}(\mathscr{P})$.

Proof. Suppose that the assertion is false. Then there is a least ordinal $\lambda$ (which is obviously a limit ordinal) and a set $K$ for which it is false. As $\lambda<\omega_{1}$, there is an increasing sequence, $\left\{\lambda_{n}: n=1,2, \ldots\right\}$ converging to $\lambda$. However, $\chi_{K \cap W_{\lambda_{n}}} \in$ $B_{1}(\mathscr{P})$ for each $n$, and so Theorem 3.5 guarantees that $\chi_{K \cap W} \in B_{1}(\mathscr{P})$ and this contradicts the choice of $\lambda$ and $K$.

Lemma 3.7. Suppose that $\chi_{K} \in \mathbb{Q} \mathscr{P}_{1}$ and that $W$ is an open set. Then $\chi_{K} \cap W \in$ $Q \mathscr{P}_{1}$.

Proof. Suppose that $K_{1}=K \cap W$ and that $\chi_{K_{1}} \notin \mathscr{Q} \mathscr{P}_{1}$. Then there exist nonempty sets $U$ and $V$ such that $U \subset K_{1}^{c}$ and $V \subset K_{1}$ such that $U \cup V \subset$ $\Delta(\bar{U} \cap \bar{V})$. Now $V \subset K_{1} \subset K$, and we shall show that $U \subset K^{c}$, and this will contradict the supposition that $\chi_{K} \in Q \mathscr{P}_{1}$. To see that $U \subset K^{c}$, consider $x \in U$, so $x \in \Delta(\bar{U} \cap \bar{V}) \subset \Delta \bar{V} \subset \bar{V}$. Now $\bar{V} \cap W=\varnothing$, thus, since $x \notin K_{1}, x \notin K$.

THEOREM 3.8. If $\chi_{K} \in \mathscr{Q} \mathscr{P}_{1}$, then $\chi_{K} \in B_{1}(\mathscr{P})$.

Proof. Suppose that $\chi_{K} \in \mathbb{Q} \mathscr{P}_{1}$. Inductively, we let $K_{0}=K$, and assuming that $K_{\alpha}$ is defined for $\alpha<\beta$, we let $G_{\alpha}$ and $H_{\alpha}$ respectively denote the maximal open sets missing $\Delta \bar{K}_{\alpha} \backslash K_{\alpha}$ and $\Delta \bar{K}_{\alpha} \cap K_{\alpha}$. We then define $K_{\beta}=K \backslash \cup_{\alpha<\beta}\left(G \cup H_{\alpha}\right)$. Let $\lambda$ be the least countable ordinal such that $\left|\bar{K}_{\lambda}\right|=0$. (The guarantee of the existence of $\lambda$ comes from Theorem 3.4.) Since $\chi_{K_{\lambda}}$ obviously belongs to $B_{1}(\mathscr{P})$, we consider $\chi_{K \backslash K_{\lambda}}$. For $\alpha<\lambda$, let $W_{\alpha}=G_{\alpha} \cup H_{\alpha}$, and let $W=\cup_{\alpha<\lambda} W_{\alpha}$. As $\chi_{K \cap W}$ $=\chi_{K \backslash K_{\lambda}}$, by Corollary 3.6, we need only to show that $\chi_{K \cap W_{\alpha}} \in B_{1}(\mathscr{P})$ for each $\alpha<\lambda$.

Suppose there exists $\alpha<\lambda$ such that $\chi_{K \cap W_{\alpha}} \notin B_{1}(\mathscr{P})$. Then there exists a first ordinal, $\beta$, such that $\chi_{K \cap W_{\beta}} \notin B_{1}(\mathscr{P})$, and as $K \cap W_{0}=K_{0} \cap W_{0}, \beta>0$ (see Theorem 3.3). Now let $W^{\prime}=\cup_{\alpha<\beta} W_{\alpha}$. It follows from Theorem 3.3 and Corollary 3.6 that both $\chi_{K_{\beta} \cap W_{\beta}}$ and $\chi_{K \cap W^{\prime}}$ are in $B_{1}(\mathscr{P})$. Thus, $\chi_{K \cap W_{\beta}}=$ $\max \left\{\chi_{K_{\beta} \cap W_{\beta}}, \chi_{K \cap W^{\prime}}\right\}$ is in $B_{1}(\mathscr{P})$, a contradiction. Finally, we notice that $\chi_{K}=$ $\max \left\{\chi_{K_{\lambda}}, \chi_{K \backslash K_{\lambda}}\right\}$ is in $B_{1}(\mathscr{P})$, and the proof is complete.

This, of course, is the desired result, but for characteristic functions. In order to extend the scope of Theorem 3.8, we first verify an analogous result for simple functions and then extend to the full case using the results from $\S 2$.

LEMMA 3.9. If $f$ is a simple function and $f \in Q^{P_{1}}$, then if $y$ is a number and $K=f^{-1}(y), \chi_{K} \in \mathbb{Q} \mathscr{P}_{1}$.

Proof. This is trivial unless $K \neq \varnothing$. Assuming that $K \neq \varnothing$ but that $\chi_{K} \notin \mathbb{Q} \mathscr{P}_{1}$, there exist $\varnothing \neq U \subset K^{c}$ and $\varnothing \neq V \subset K$ such that $U \cup V \subset \Delta(\bar{U} \cap \bar{V})$. Let $A=U \cap\{x: f(x)>y\}$ and $B=U \cap\{x: f(x)<y\}$. Now, there exists a nonempty relatively open subset $I$ of $\bar{U} \cap \bar{V}$ such that either $A$ is dense in $I$, or $B$ is dense in $I$.

In the first case, choose $y<a<b$ such that $\{x: y<f(x) \leqslant b\}=\varnothing$; in the second case, choose $a<b<y$ such that $\{x: a \leqslant f(x)<y\}=\varnothing$. For the first case, 
let $\varnothing \neq U^{\prime}=V \cap I$ and $\varnothing \neq V^{\prime}=A \cap I$. For the second case, let $\varnothing \neq U^{\prime}=$ $B \cap I$ and $\varnothing \neq V^{\prime}=V \cap I$. In either case, $U^{\prime} \cup V^{\prime} \subset \Delta\left(\bar{U}^{\prime} \cap \bar{V}^{\prime}\right)$, contradicting the assumption that $f \in \mathbb{Q} \mathscr{P}_{1}$.

Combining Lemma 2.2, Lemma 2.6, . heorem 3.8 and Lemma 3.9, plus the fact that a bounded simple function can be expressed as $\sum_{i=1}^{n} c_{i} \cdot \chi_{K_{i}}$, we get the following.

Corollary 3.10. If $f$ is bounded and $f \in \mathscr{Q}^{\mathscr{P}_{1}}$, then $f \in B_{1}(\mathscr{P})$.

In order to extend Corollary 3.10 to include the unbounded case, we make the following observation.

LEMMA 3.11. The function $f \in B_{1}(\mathscr{P})$ if and only if $\operatorname{Arctan}(f) \in B_{1}(\mathcal{P})$. Also, $f \in \mathbb{Q} \mathscr{P}_{1}$ if and only if $\operatorname{Arctan}(f) \in \mathbb{Q} \mathscr{P}_{1}$.

THEOREM 3.12. If $f \in \mathbb{Q} \mathscr{P}_{1}$, then $f \in B_{1}(\mathscr{P})$.

Proof. If $f \in \mathbb{Q} \mathscr{P}_{1}$, then $\operatorname{Arctan}(f) \in \mathbb{Q} \mathscr{P}_{1}$. But then Corollary 3.10 implies that $\operatorname{Arctan}(f) \in B_{1}(\mathscr{P})$, and from this it follows that $f \in B_{1}(\mathscr{P})$.

\section{BIBLIOGRAPHY}

[CH] G. Cox and P. Humke, A note on pointwise limits of functions which are approximately continuous and almost everywhere continuous, Studia Sci. Math. Hungar. (to appear).

[GW] C. Goffman and D. Waterman, Approximately continuous transformations, Proc. Amer. Math. Soc. 12 (1961), 116-121.

[G] Z. Grande, Sur les suites de fonctions approximativement continues et continues presque partout, Colloq. Math. 38 (1978), 259-262.

[K] K. Kuratowski, Topology. I, Academic Press, New York, 1966 and PWN, Warsaw, 1958.

[M] D. Mauldin, o-ideals and related Baire systems, Fund. Math. 71 (1971), 171-177.

[N] T. Nishiura, The topology of almost everywhere continuous, approximately continuous functions, Acta Math. (to appear).

[O1] R. J. O’Malley, Approximately differentiable functions: the $r$ topology, Pacific J. Math. 72 (1977), 207-222.

$\left[\mathrm{O}_{2}\right] \ldots$, Approximately continuous functions which are continuous almost everywhere, Acta Math. Acad. Sci. Hungar. 33 (1979), 395-402.

[P] D. Preiss, Limits of approximately continuous functions, Czechoslovak Math. J. 96 (1971), 371-372.

Department of Mathematics, Western Illinois University, Macomb, ILIINOIS 61455

Current address (G. V. Cox): T. R. W. Systems, P. O. Box 1310, San Bernardino, California 92402

Current address (P. D. Humke): Department of Mathematics, St. Olaf College, Northfield, Minnesota 55057 